\title{
Every Set Has at Least Three Choice Functions
}

\author{
Milan R. TASKOViĆ
}

\begin{abstract}
This paper continues the study of the Axiom of Choice by E. Z e r m elo [Neuer Beweis für die Möglichkeit einer Wohlordung, Math. Annalen, 65 (1908), 107-128; translated in van Heijenoort 1967, 183-198]. We prove some new equivalents of the Axiom of Choice, i.e., Zorn's lemma, and in connection with an initial equivalent also fact that every set has at least three choice functions.
\end{abstract}

\section{History And AnNotations}

We shall first discuss an assumption that appears to be independent of, and yet consistent with, the usual logical assumptions regarding classes and correspondences, but whose absolute validity has been seriously questioned by many authors. This is the so-called Axiom of Choice, which has excited more controversy than any other axiom of set theory since its formulation by Ernst Zermelo in 1908. In this sense, many results are known in the set theory.

In 1904 , Zermelo $^{1}$ stated a principle of choice similar to: If $\mathcal{D}$ is a family of nonempty sets, there is a function $f$ such that $f(A) \in A$ for every $A \in \mathcal{D}$; and proved that it implied the well-ordering theorem. In 1908 Zermelo proposed main version of the Axiom of Choice. This is the connection and with a conversations with Erhardt Schmidt.

Bertrand Russell in 1906 gave a principle analogous to preceding. He announced this principle as a possible substitute for Zermelo's but he believed that it was weaker. Zermelo, in 1908 stated and, proved that Russell's and his formulations of the axiom of choice are equivalent. The name "axiom of choice" is due to Zermelo in 1904.

Apparently, the first specific reference to the axiom of choice was given in a paper by G. Peano ${ }^{2}$ in 1890 . In proving an existence theorem for ordinary

2000 Mathematics Subject Classification. Primary: 47H10; Secondary: 54H25.

${ }^{1} \mathrm{~B}$ e f or e 1904 , when $\mathrm{Z}$ e r m e lo published his proof that the axiom of choice implies the well-ordering theorem, the well-ordering theorem was considered as self-evident. $\mathrm{C}$ ant or and the others used it without hesitation.

${ }^{2} \mathrm{G}$ i u s e p p e P e a n o: "But as one cannot apply infinitely many times an arbitrary rule by which one assigns to a class $A$ an individual of this class, a determinate rule is stated here". 
differential equations, he ran across a situation in which such a statement is needed. Beppo Levi in 1902, while discussing the statement that the union of a disjoint set $S$ of nonempty sets has a cardinal number greater than or equal to the cardinal number of $S$, remarked that its proof depended on the possibility of selecting a single member from each element of $S$. Others, including Georg Cantor, had used the principle earlier, but did not mention it specifically.

In this time, the Axiom of Choice asserts that for every set $S$ there is a function $f$ which associates each nonempty subset $A$ of $S$ with a unique member $f(A)$ of $A$. From a psychological perspectie, one might express the Axiom by saying that on element is "chosen" from each subset $A$ of $S$. However, if $S$ is infinite, it is difficult to conceive how to make such choices - unless a rule is available to specify an element in each $A$.

David Hilbert, in 1926, once wrote that Zermelo's Axiom of Choice ${ }^{3}$ was the axiom "most attacked up to the present in the mathematical literature ..."; to this, Abraham Fraenkel later added that "the axiom of choice is probably the most interesting and, in spite of its late appearance, the most discussed axiom of mathematics, second only to Euclid's axiom of parallels which was introduced more than two thousand years ago".

The equivalence of the axiom of choice and the trichotomy was given by Hartogs in 1915. As in the case of the well-ordering theorem, the trichotomy was considered self-evident and was used without hesitation before 1915 .

As mathematics developed futher there also developed a need for another non-constructive proposition; a principle, which Kuratowski, Hausdorff, Zorn, and others, used to replace transfinite induction and the well-ordering theorem. It appears, at first glance, unrelated to the axiom of choice, but actually is equivalent to it.

This principle and principles similar to it are often referred to as forms of Zorn's lemma. In 1933 Artin and Chevalley first referred to the principle as Zorn's lemma.

3ZZermelo's Reply to His Critics. During the summer of 1907 Z e r m e lo took stock of the criticisms directed against both his Axiom and his proof of the well-ordering theorem. One in 1908 was a reply to his critics, and the other also in 1908 contained the first axiomatization of set theory. Zermelo's first article in 1908 began with a new demonstration of the well-ordering theorem.

From them he developed the properties of his $\theta$-chains, which generalized Dedekind's earlier concept of chain. Z e r m e lo corresponded with J o u r d a in in 1907, but apparently their letters focused on a generalization of König's theorem.

Although he had read Borel's article and the published correspondence between: $\mathrm{B}$ a i r e, B or e l, $\mathrm{H}$ a d a $\mathrm{m}$ a r d, and $\mathrm{L}$ e b e s g u e, he concetrated on refuting $\mathrm{P}$ e a $\mathrm{n}$ o with whom he had previously feuded over the equivalence theorem.

During 1906 he corresponded with $\mathrm{P}$ o i n c a r é regarding his proof and his axiomatization of set theory. A letter, as well as three others from Poincaré, is kept in Zermelo's Nachlass at the University of Freiburg in Breisgau. De facto, Z e r m e lo emerged as a realist in much situations, perhaps a Platonist!? 
The history of maximal principles is quite tangled. The earliest reference to a maximal principle in the literature is in 1907 from Hausdorff.

In 1910 independently Janiszewski, Mazurkiewicz and Zoretti published a special case Hausdorff's principle in the form of a theorem in topology. In 1905 Lindelöf, in 1911 Brouwer, and in 1920 Sierpiński derivated some more general topological theorems from the well-ordering theorem.

In 1922 Kuratowski derived minimal principles equivalent to the preceding principles from the well-ordering theorem. Kuratowski in 1922 used a minimal principle to prove a theorem in analysis, as and R.L. Moore in 1932 .

In set theory, we notice that, all of the usual mathematical concepts can be reduced to the notion of set.

The mathematical concept of a set can be used as the foundation for all known mathematics facts. A flock of pigeons, or a bunch of grapes are examples of sets of things. Sets, as they are usually conceived, have elements or members. An element of a set may be a pigeon, or a grape; i.e., this means an atomistic classical admission sets. It is important to know that a set itself may also be an element of some other set. Mathematics is full of examples of sets of sets.

Within the Cantorian tradition, one can view Zermelo's axiomatization as answering the question: What is a set?! This question has served as a theme in the development of set theory, but one not often discussed openly.

In the meanthime, there has developed a concept of the set-theory damaged school child, so we must ensure that this paradise remains a blooming garden and does not turn into rocky ground and thorny scrub. In this sense, our admission, for this problem in this paper, give a new paradise for set-theory.

The classes of objects encountered in the real or a concoction physical word do not have precisely defined conditions of membership. In 1965 Zadeh introduced the notion of a fuzzy set which is an answer on this indistinction. A fuzzy set is a class of objects with a continuum of grades of membership.

In connection with the preceding, in this section we introduce the concept of a transversal set as a natural extension of ordinary, fuzzy and transversal (upper or lower) fuzzy sets. A transversal set is a class of objects with two characteristics: first, with a continuum of grades of membership and second, with new signification (an annex) foreign or inside influence and affair.

Let $X$ be a nonempty set, let $L:=(L, \preccurlyeq)$ be a lattice (or a complete lattice) by the order relation $\preccurlyeq$ and let $g$ be a given mapping from $L$ into $L$. A transversal upper set $A$ (or an upper side of $A$ in notation $A_{u s}$ ) in $X$ is characterized by a transversal membership (characteristic) function

$$
\sup \left\{f_{A}(x), g\left(f_{A}(x)\right\}: X \rightarrow L,\right.
$$

where $f_{A}(x): X \rightarrow L$ is a set function of $A$. In this case, the value of $f_{A}(x)$ at $x \in X$ can be representing the grade of membership of $x$ in $A$, till the 
value of $g\left(f_{A}(x)\right)$ can be representing an event (foreign or inside) which is in connection with the set $A$ in $X$. Precisely, $A_{u s}$ is completely characterized by the set of pairs in the form as

$$
A_{u s}:=\left\{\left(\sup \left\{f_{A}(x), g\left(f_{A}(x)\right)\right\}, x\right): x \in X\right\} .
$$

In connection with this, let $P:=(P, \preccurlyeq)$ be a partially ordered set and for $a, b \in P$ and $a \preccurlyeq b$, the set (interval) $[a, b]$ is defined by

$$
[a, b]:=\{t: t \in P \text { and } a \preccurlyeq t \preccurlyeq b\} .
$$

If the lattice $L:=[a, b]$ for $a \preccurlyeq b$, then we have a very typical example of the transversal membership characteristic function (1). A special case, in this sense, if $[a, b] \subset \mathbb{R}(a<b ; a, b \in \mathbb{R})$ is essential for further applications. Also, if $[a, b]=[0,1]$, then (1) reduces to the membership characteristic function which representing the transversal (upper) fuzzy set $A$ in $X$, which is introduced in Tasković [2004].

Example 1. Let $X$ be the real line $\mathbb{R}$ and let $A$ be a transversal upper set of numbers which are much greater than 1 . Then for $g: I \rightarrow I$ defined by $g(x)=1-x$ and $f_{A}(0)=1, f_{A}(1)=0, f_{A}(4)=0.02, f_{A}(10)=0.03, f_{A}(99)=0.81$ and $f_{A}(400)=0.93$, we obtain a characterization of the set $A$ in $\mathbb{R}$.

In analogous with the preceding facts, let $X$ be a nonempty set, let $L:=$ ( $L, \preccurlyeq)$ be a lattice (or a complete lattice) and let $d$ be a given mapping from $L$ into $L$. A transversal lower set $A$ (or a lower side of $A$ in notation $A_{l s}$ ) in $X$ is characterized by a transversal membership (characteristic) function

$$
\inf \left\{f_{A}(x), d\left(f_{A}(x)\right)\right\}: X \rightarrow L,
$$

where $f_{A}(x): X \rightarrow L$ is a set function of $A$. Precisely, $A_{l s}$ is completely characterized by the set of pairs in the form as

$$
A_{l s}:=\left\{\left(\inf \left\{f_{A}(x), g\left(f_{A}(x)\right)\right\}, x\right): x \in X\right\} .
$$

A transversal set $A$ (or a medial side of $A$ in notation $A_{m s}$ ) in $X$ is a transversal upper and lower set, simultaneous. As an important example of transversal sets we have a Zadeh's fuzzy set in the case $g(x)=x: I \rightarrow I$.

If the lattice $L:=[a, b]$ for $a \preccurlyeq b$, then we have a very typical example of the transversal membership characteristic function (2). A special case, in this sense, if $[a, b] \subset \mathbb{R}(a<b ; a, b \in \mathbb{R})$ is essential for further applications. Also, if $[a, b]=[0,1]$, then (2) reduces to the membership characteristic function which representing the transversal (lower) fuzzy set $A$ in $X$, which is introduced in Tasković [2004].

Some remarks. In the case new situations, a transversal (upper or lower) set is obviously an extension of an ordinary and a fuzzy set, and the 
transversal membership (characteristic) functions is a generalization of the membership functions of the ordinary and of the fuzzy set! $!^{4}$

But our new concept of transversal sets (1) and (2) give manyfold and have an ambiguous in looking of the sets and in looking of the new calculation with them!

We notice, the transversal characteristic membership functions given in formulas (1) and (2) are not as the "standard" union and intersection, respectively, of the membership functions for the ordinary and the fuzzy sets!

The transversal (characteristic) membership functions (1) and (2), respectively, are essential different of the union and intersection fot the ordinary and the fuzzy sets!

We notice that, from the former facts in Taskovic [2004], we give a technology of an arbitrary set (as atom) via transversal upper and lower sets.

In this sense, every set has three part (or three sides, or three projections) as a bell, or as a coin.

Every set has three microscopic projections as parts which are not see, but this sides existing as three (upper, lower and medial) transversal sets.

Every atom (particle) in physics can be explain as a transversal set which has: positrons (as an upper transversal set), electrons (as a lower transversal set), and neutrons (as a transversal set). This are new facts on atoms in physics as a new realism.

The preceding "three sides" of an arbitrary set to means adequate that there exist three transversal integrals (upper, lower, and medial). This is an explanation that existing, an example, three Riemann's integrals.

We notice that, de facto, in Tasković [2004] is presents a new concept of set which we call it transversal (upper, lower or medial) set. ${ }^{5}$ We introduce this concept as a natural extension of ordinary, fuzzy and transversal fuzzy sets. Transversal sets are a new way in the nonlinear analysis. First time in history of the theory sets, we give a technology of an arbitrary set, in the sense that every set has "three sides" which are invisible but they de facto existing by Tasković [2004].

In connection with the preceding, let $X$ be a nonempty set, let $L:=(L, \preccurlyeq)$ be a lattice (or a complete lattice) and let $g: L^{k} \rightarrow L$ be a given map, $k$ is a fixed positive integer, i.e., $k \in \mathbb{N}$. An $k$-transversal upper set $A$

\footnotetext{
${ }^{4}$ In this sense, we give a technology of an arbitrary set such that, de facto, every set has three sides which are invisible but they existing. Also, in a text of $\mathrm{R} \mathrm{i} \mathrm{c} \mathrm{h} \mathrm{a} \mathrm{r} \mathrm{d}$ D e d e k i n d (1831-1916) spell: "I think of a set as a closed sack which contains certain specified objects which one doesn't see".

${ }^{5} \mathrm{Georg}$ Cantor: I think of a set as a precipice. On the other hand, Leopold Kronecker brief: Cantor is the corruptor of youth. Richard Dedekind: "I think of a set as a closed sack which contains certain specified objects which one doesn't see". David Hilbert in 1925: "No one should ever drive us from the paradise which Cantor created for us". Bertrand Russel: "Thus mathematics may defined as the subject in which we never know that we are talking about, nor whether what we are saying is true".
} 
(or an upper transversal set $A$ is $k$-dimension) in $X$ is characterized by a transversal membership (characteristic) function

$$
\sup \left\{f_{A}\left(x_{1}\right), \ldots, f_{A}\left(x_{k}\right), g\left(f_{A}\left(x_{1}\right), \ldots, f_{A}\left(x_{k}\right)\right)\right\}: X^{k} \rightarrow L,
$$

where $f_{A}\left(x_{1}\right), \ldots, f_{A}\left(x_{k}\right): X \rightarrow L$ is a set function of $A$. If $k=1$, then we have the case of a transversal upper set $A$ in $X$.

Let $X$ be a nonempty set, let $L:=(L, \preccurlyeq)$ be a lattice (or a complete lattice) and let $d: L^{k} \rightarrow L$ be a given map, where $k \in \mathbb{N}$ is fixed. An $k$-transversal lower set $A$ (or a lower transversal set $A$ is $k$-dimension) in $X$ is characterized by a transversal membership (characteristic) function

$$
\inf \left\{f_{A}\left(x_{1}\right), \ldots, f_{A}\left(x_{k}\right), d\left(f_{A}\left(x_{1}\right), \ldots, f_{A}\left(x_{k}\right)\right)\right\}: X^{k} \rightarrow L,
$$

where $f_{A}\left(x_{1}\right), \ldots, f_{A}\left(x_{k}\right): X \rightarrow L$ is a set function of $A$. If, in this case, $k=1$, then we obtain the case of a transversal lower set $A$ in $X$.

In connection with this, we notice that, in Tasković [2004], applications in minimax theory, algebra, topology, analysis, games theory, algebraic equations theory, ideal theory of BCC-algebras, theory of measures and integration, and convex analysis are considered.

Main annotation. From the preceding and the former facts, we obtain that every set, de facto, has three sides (in the preceding sense), and that every side can be of $k$-dimension!

\section{New equivalents of Axiom of Choice}

In general, equivalents of the Axiom of Choice appear frequently in almost all branches of mathematics in a large variety of different forms.

In this part of paper we present some new equivalent forms of Axiom of Choice which are expressible in the following sense.

Theorem 1 (Axiom of Choice). Let $S$ be an arbitrary set. Then the following statement are equivalent:

(a) (Zermelo's Principle). Given any set $S$, there is a function $f$ such that for each nonempty subset $A$ of $S, f(A) \in A$.

(b) (Axiom of 3-Choice). Given any set $S$, there exist at least three functions $f_{k}(k=1,2,3)$ such that for each nonempty subset $A$ of $S$, $f_{k}(A) \in A$ as $k=1,2,3$.

(c) If $S$ is a set, $T=\mathcal{P}(S) \backslash\{\varnothing\}$ where $\mathcal{P}$ is the power class, $F$ is the set of all functions from $T$ to $S$ and $g$ is a function from $F$ to $T$, then there exist at lest three functions $f_{k} \in F(k=1,2,3)$ such that $f_{k}\left(g\left(f_{k}\right)\right) \in g\left(f_{k}\right)$ for $k=1,2,3$.

Proof. Let (a) holds and let $S$ be a given nonempty set. Since, from the preceding facts of first part, the set $S$ has "three sides" applying (a) on each of these sides we obtain three choice functions $f_{k}(k=1,2,3)$. Then $f_{k}$ $(k=1,2,3)$ are the required functions, i.e., (b) holds. 
For proof that (b) implies (c) let $S, T=\mathcal{P}(S) \backslash\{\varnothing\}, F$ and $g$ satisfy the hypothesis of $(\mathrm{c})$. Then if $f_{k}(k=1,2,3)$ are choice functions on $T$, then $f_{k} \in F, g\left(f_{k}\right) \in T$ and $f_{k}\left(g\left(f_{k}\right)\right) \in g\left(f_{k}\right)$ for $k=1,2,3$.

This means that (c) is a consequence of (b). Thus, we need only show that (c) implies (a).

Suppose (a) is false. Then there is a set $S$ such that if $T=\mathcal{P}(S) \backslash\{\varnothing\}$, and $F$ is the set of all functions mapping $T$ into $S$ then for all $f \in F$ there is a $U \in T$ such that $f(U) \notin U$. Suppose $p \notin S$. For each ordinal $\alpha$ and for each $f \in F$, define

$$
\psi_{f}(\alpha):= \begin{cases}f(S \backslash \operatorname{im} f), & \text { if } f(S \backslash \operatorname{im} f) \in S \backslash \operatorname{im} f \\ p, & \text { otherwise; }\end{cases}
$$

where $\operatorname{im} f$ is image of the mapping $\psi_{f}(\alpha)$. Since $\psi_{f}^{-1}$ is a bijection, thus there is an $\alpha$ such that $\psi_{f}(\alpha)=p$. Let $\alpha_{0}$ be the smallest such $\alpha$. If $\operatorname{im} f\left(\alpha_{0}\right)=S$, then $S$ can be well ordered, which implies $T$ has a choice function, contradicting our assumption. Thus, $\operatorname{im} f\left(\alpha_{0}\right) \subset S$. Define that $g(f)=S \backslash \operatorname{im} f\left(\alpha_{0}\right)$. Then $g$ is a function with domain $F$, range contained in $T$ and $f(g(f)) \notin g(f)$ for all $f \in F$. This contradicts (c). The proof is complete.

Theorem 2 (Restatements of Axiom of 3-Choice). Let $S$ be an arbitrary set and let $\mathcal{D}$ denoted domain and $\mathcal{R}$ denoted range. Then the following statements are equivalent:

(a) (Axiom of 3-Choice). Given any set $S$, there exist at least three functions $f_{k}(k=1,2,3)$ such that for each nonempty subset $A$ of $S$, $f_{k}(A) \in A$ as $k=1,2,3$.

(b) For every function $f$ there exist three functions $f_{k}(k=1,2,3)$ such that for every $x$, if $x \in \mathcal{D}(f)$ and $f(x) \neq \varnothing$, then $f_{k}(x) \in f(x)$ as $k=1,2,3$.

(c) For every relation $r$ there exists three functions $f_{k}(k=1,2,3)$ such that $\mathcal{D}\left(f_{k}\right)=\mathcal{D}(r)$ and $f_{k} \subset r$ as $k=1,2,3$.

(d) For every function $f$ there exist three functions $f_{k}(k=1,2,3)$ such that $\mathcal{D}\left(f_{k}\right)=\mathcal{R}(f)$ and for every $x \in \mathcal{D}\left(f_{k}\right)$ is $f\left(f_{k}(x)\right)=x$ as $k=1,2,3$.

Proof. We shall first show that (a) implies (b). Let $f$ be an arbitrary function. Let $S=\mathcal{R}(f)$ and let $F_{k}(k=1,2,3)$ be choice functions on $S$. Define functions $f_{k}(k=1,2,3)$ such that for each $x \in \mathcal{D}(f), f_{k}(x)=F_{k}(f(x))$. Then $f_{k}(k=1,2,3)$ are the required functions.

Also, (b) implies (a). In this sense, let $\mathcal{L}$ be a set of nonempty sets of $S$. Let $f$ be a bijection function such that $\mathcal{R}(f)=\mathcal{L}$. Define three functions $F_{k}$ $(k=1,2,3)$ such that for each $x \in \mathcal{L}$ we have $F_{k}(x)=f_{k}\left(f^{-1}(x)\right)$, where $f_{k}(k=1,2,3)$ are defined by (b). Then $F_{k}(k=1,2,3)$ are the required choice functions. 
For proof that (c) implies (d), let $f$ be an arbitrary function and let $r=\{(x, y):(y, x) \in f\}$. Then, (c) implies that there exist functions $f_{k}$ $(k=1,2,3)$ such that $\mathcal{D}\left(f_{k}\right)=\mathcal{D}(r)$ and $f_{k} \subset r$ as $k=1,2,3$. Clearly, for every $x \in \mathcal{R}(f)=\mathcal{R}\left(f_{k}\right)$ we have $f\left(f_{k}(x)\right)=x$ as $k=1,2,3$.

Also, (d) implies (c). Indeed, let $r$ be an arbitrary relation and define a function $h$ as follows that $h=\{((x, y), x):(x, y) \in r\}$. Then (d) implies that there exist functions $F_{k}(k=1,2,3)$ such that $\mathcal{D}\left(F_{k}\right)=\mathcal{D}(h)$ and for every $x \in \mathcal{D}\left(F_{k}\right)$ we have $h\left(F_{k}(x)\right)=x$ as $k=1,2,3$. Now, $F_{k}(k=1,2,3)$ are ordered pairs, so we define $f_{k}(x)$ as $k=1,2,3$ to be the second coordinate of $F_{k}(k=1,2,3)$ for each $x \in \mathcal{D}\left(F_{k}\right)=\mathcal{D}(r)$ as $k=1,2,3$. Clearly, $\mathcal{D}\left(f_{k}\right)=\mathcal{D}(r), f_{k}(k=1,2,3)$ are functions, and $f_{k} \subset r$ as $k=1,2,3$.

In connection with this, (c) implies (b), also. Indeed, let $f$ be an arbitrary function. Define a relation $r$ as follows: $r=\{(x, y): y \in f(x)\}$. Then, (c) implies that there exist functions $f_{k}(k=1,2,3)$ such that $\mathcal{D}\left(f_{k}\right)=\mathcal{D}(r)$ and $f_{k} \subset r$ as $k=1,2,3$. In this case, $f_{k}(k=1,2,3)$ are the required functions.

Also, (b) implies (c). In this sense, let $r$ be an arbitrary relation. Define a function $h$ as follows: $h(x)=\{y:(x, y) \in r\}$ for $x \in \mathcal{D}(r)$. Also, (b) implies that there exist functions $f_{k}(k=1,2,3)$ such that if $x \in \mathcal{D}(h)$ and $h(x) \neq \varnothing$, then $f_{k}(x) \in h(x)$ as $k=1,2,3$. Then, $f_{k}(k=1,2,3)$ are the required functions. Now, the proof is complete.

In connection with the preceding facts, research continued on the deductive strength of various statements relative to the Boolean Prime Ideal Theorem. In 1961 Jan Mycielski considered the following statement $P_{n}$ for each $n>1$ in form: If $G$ is a graph such that every finite subgraph of $G$ can be colored with $n$ colors, then $G$ itself can be colored with $n$ colors. Höft and Howard later, in 1973, gave a graph-theoretic equivalent of the Axiom of Choice.

In 1955 W. Kinna and K. Wagner, introduced a different kind of proposition as a weakening of the Axiom of Choice: For every set $S$ there is a function $f$ such that, for each subset $A$ of $S$ with two or more elements, $f(A)$ is a nonempty proper subset of $A$.

Also, in $1962 \mathrm{~A}$. Levy considered the following propositions $Z(n)$, closely related to the Kinna-Wagner Principle in form: For every family $\mathcal{F}$ of nonempty sets there is a function $f$ such that, for each $A$ in $\mathcal{F}, f(A)$, is a nonempty subset of $A$ having at most $n$ elements.

In connection with the preceding facts we have the following result which is a consequence of the preceding two statements.

Theorem 3 (Axiom of 3-Choice). Let $S$ be an arbitrary set. Then the following statements are equivalent:

(a) Let $m \geq 1$ be a natural number. For every set $S$ there exists an ordinal number $\alpha$ and three functions $f_{k}(k=1,2,3)$ defined on 
$\alpha$ such that $f_{k}(\beta) \preccurlyeq m$ for every $\beta<\alpha$ and $\cup_{\beta<\alpha} f_{k}(\beta)=S$ as $k=1,2,3$.

(b) For every set $S$ there exist a natural number $m \geq 1$, an ordinal number $\alpha$, and three functions $f_{k}(k=1,2,3)$ defined on $\alpha$ such that $f_{k}(\beta) \preccurlyeq m$ for every $\beta<\alpha$ and $\cup_{\beta<\alpha} f_{k}(\beta)=S$ as $k=1,2,3$.

(c) Let $m \geq 1$ be a natural number. If $\mathcal{F}$ is a set of nonempty sets there exist three functions $f_{k}(k=1,2,3)$ such that for each $A \in \mathcal{F}, f_{k}(A)$ is a nonempty subset of $A$ with $f_{k}(A) \preccurlyeq m$ as $k=1,2,3$.

(d) If $\mathcal{F}$ is a set of nonempty sets then there exist a natural number $m \geq 1$ and three functions $f_{k}(k=1,2,3)$ such that for each $A \in \mathcal{F}$, $f_{k}(A)$ is a nonempty subset of $A$ with $f_{k}(A) \preccurlyeq m$ as $k=1,2,3$.

We notice that the following implications are clear and a totally analogy with the preceding proofs: (a) is equivalent to the (b). Also, (c) and (d) are consequences of (b). Thus, we need only show that (d) implies (b).

In this sense, let $A$ be a set and let $\mathcal{F}$ be the set of all nonempty subsets of $A$. By (d), there exist a natural number $m \geq 1$ and three functions $F_{k}(k=1,2,3)$ such that for each $A \in \mathcal{F}, F_{k}(A) \neq \varnothing, F_{k}(A) \subset A$, and $F_{k}(A) \preccurlyeq m$ for $k=1,2,3$.

Define $F_{k}(\varnothing)=u$, where $u \notin A$. Define the functions $G_{k}(k=1,2,3)$ as follows: For all ordinal numbers $\alpha, G_{k}(\alpha)=F_{k}\left(A \backslash \cup_{\beta<\alpha} G_{k}(\beta)\right)$. Now, we have that $G_{k}^{-1}$ is bijective on $\mathcal{R}\left(G_{k}\right) \cap \mathcal{R}(A)$, and there is an ordinal $\alpha$ such that $\cup \operatorname{im} G_{k}(\alpha)=A$. Then define $f_{k}=G_{k} \mid \alpha$, and we have $m \geq 1$ is the natural number, $\alpha$ is the ordinal number and $f_{k}(k=1,2,3)$ are the functions which satisfies (b). The proof is complete.

It wasn't until 1935 that Max Zorn ${ }^{6}$ published his paper. He was the first one to use a maximal principle in algebra. He stated without proof that this the maximal principle is equivalent to the axiom of choice. For this proof Zorn credits Artin and Kuratowski.

In France, where the Axiom had been so poorly received three decades earlier, Zorn's friend Chevalley introduced the maximum principle to the Bourbakists and after dedicing, Bourbaki stated Zorn's principle as a corollary.

In 1940, also influenced by Zorn, the Princeton topologist John Tukey deduced from the Axiom four variants of what he termed Zorn's lemma, and sketched a proof of their equivalence to the Axiom of Choice.

\footnotetext{
${ }^{6}$ What were the beginnings of Zorn's principle? According to his later reminiscences, he first formulated it at Hamburg in 1933, where Claude Chevalley and Emil Artin then took it up as well. Indeed, when $\mathrm{Z}$ o r n applied it to obtain representatives from certain equivalence classes on a group, A r t i n recognized that Zorn's principle yields the Axiom of Choice. By late in 1934, Zorn's principle had found users in the United States who dubbed in Zorn's lemma. In October, when Zorn lectured on his principle to the American Mathematical Society in New York, S ol o m on L e f s chetz recomended that Zorn publish his result. The paper appeared, the following year, in 1935.
} 
Nevertheless, there remained one final independent rediscovery, due to the German algebraist O. Teichmüller then in 1939 a Privatdozent at Berlin. This principle is often referred to as form of Teichmüller-Tukey lemma.

The Serbian mathematician Djuro Kurepa found in 1952 a number of relations $R$ such that the corresponding maximal principle was an equivalent.

In 1960 two American mathematicians, Herman and Jean Rubin, were prompted by Kurepa's research to consider maximal principles. In addition, $\mathrm{H}$. Rubin found two statements which were equivalent to the Axiom of Choice in $Z F$, but were weaker in $Z F U$. In 1963 the Rubins published a book summarizing and completing much of the earlier work on equivalents.

On the other hand, in 1936 the American mathematician Marshall Stone, then at Harvard, contributed his influential findings on the representation of Boolean rings. Stone deduced a proposition equivalent to it and later known as the Stone Representation Theorem.

In 1939 A. Tarski was studying the number of prime ideals found in rings of sets. Later, in 1940, Birkohoff observed that his representation theorem for distributive lattices had been inspired by the researches of Tarski.

Probably the most well-known and important topological equivalent of the Axiom of Choice is the Tychonoff Compactness Theorem in 1935 from a maximal principle for which in $1955 \mathrm{~J}$. Kelley proved the converse.

The second development occured on the frontier between algebra, analysis, and set theory: Stefan Banach's researches at Lwów on functional analysis.

In 1929 Banach established a fundamental result later known as the HahnBanach theorem. To obtain this result, Banach relied on the well-ordering theorem. In this sense, in analysis, the following facts are connection and hold: Krein-Milman theorem, Alaoglu's theorem, and Bell-Fremlin theorem, as and many others.

The fixpoint problem for a given mapping $f \mid P$ is very easy to formulate: the question is whether some $\zeta \in P$ satisfies $f(\zeta)=\zeta$. Many problems are reducible to the existence of fixpoints of certain mappings. The question remains whether statement (of the Axiom of Choice type) could be equivalently expressed in the fixpoint language as well. The answer is affirmative.

An existence theorem asserts the existence of an object belonging to a certain set and possessing certain properties. Many existence theorems can be formulated so that the under lying set is a partially ordered set and the crucial property is maximality.

This principle and principles similar to it are often referred to as a form of Zorn's lemma. A strong form of Zorn's lemma is due to Bourbaki. In this paper we present a new strong form of Zorn's and Bourbaki's lemma. On the other hand, we notice that a statement on the existence of maximal elements (in certain partially ordered complete subsets of a normed linear space) played a central role in the proof of the fundamental statement of 
Bishop and Phelps on the density of the set of support points of a closed convex subset of a Banach space.

The transfinite induction argument is based on Zorn's lemma. This argument was later used in a different setting by Brøndsted and Rockafellar, Browder, Ekeland, Brøndsted and others. Recently Brézis and Browder proved a very general principle concerning order relations which unifies a number of diverse results in nonlinear functional analysis.

In connection with the preceding facts we have the following results as new equivalents of the Axiom of 3-Choice.

Theorem 4 (Zorn's lemma). Let $P:=(P, \preccurlyeq)$ be a partially ordered set. Then the following statement are equivalent:

(a) (Bourbaki's lemma). Let $P$ be a quasi inductive poset, i.e., every nonempty well ordered chain in $P$ has an upper bound in $P$, then $P$ has a maximal element.

(b) Let $P$ be a quasi inductive partially ordered set. Then $P$ has at least three maximal elements.

(c) Let $P$ be a quasi inductive partially ordered set and $f$ a mapping from $P$ into $P$ such that

$$
x \preccurlyeq f(x) \text { for all } x \in \operatorname{Sub} f(P),
$$

where Sub $f(P):=f(P) \cup\{a \in P \mid a=u b C$ for some chain in $f(P)\}$ and where $u b C$ is an upper bound of $C$. Then $f$ has at least three fixed points.

(d) (Zermelo in 1908). Let $P$ be a chain complete partially ordered set, i.e., every nonempty chain in $P$ has a least upper bound in $P$, and $f$ a mapping from $P$ into itself such that: (i) there is an element $\theta \in P$ with $\theta \preccurlyeq x$ for all $x \in P$, (ii) $x \preccurlyeq f(x)$ for all $x \in P$, (iii) if $x, y \in P$ and $x \preccurlyeq y \preccurlyeq f(x)$ then either $x=y$ or $f(x) \preccurlyeq f(y)$. Then there is an element $\xi \in P$ with $f(\xi)=\xi$.

Proof. We notice that from the former facts (see: H. Rubin - J. Rubin [1970]), (a) is equivalent to Zorn's lemma. Since the set $P$ has "three sides" applying (a) on each of these sides we obtain that $P$ has at least three maximal elements, i.e., (b) holds.

Also, (b) implies (c). Indeed, by (b) there exist three maximal elements of $P$. Let $z \in P_{u s}$ a maximal element, i.e., Sub $f(P)$ has a maximal element $z \in \operatorname{Sub} f(P)$. From condition $(\mathrm{M})$ we have $z \preccurlyeq f(z)$ and, because $z$ is maximal element of the set $\operatorname{Sub} f(P), f(z) \preccurlyeq z$. Hence, $f(z)=z$, so, $f$ has a fixed point in $P$. In the second or in the third case, if $z \in P_{l s}$ or $z \in P_{m s}$, at the same as in first case we obtain that $f$ has still two fixed points. This means that (c) holds. Applying (c) to the set $\operatorname{Sub} f(P)$, we obtain that $f$ has a fixed point, i.e., we obtain that (d) holds.

Thus, we need only show that (d) implies (a), i.e., that (d) implies Zorn's lemma. In this sense, suppose that the result (a) is false. 
Then for each $x \in P$ there exists $y \in P$ with $x \preccurlyeq y$ and $x \neq y$. Let $\mathcal{T}_{0}$ be the family of all nonempty chains of $P$ and let $\mathcal{T}=\mathcal{T}_{0} \cup\{\varnothing\}$. The family $\mathcal{T}$ is partially ordered by the inclusion relation between subset of $P$. For each $A \in \mathcal{T}_{0}$ the set

$$
U_{A}=\{x \in P: x \quad \text { is an upper bound for } A \text { and } x \notin A\}
$$

is nonempty because, if $x$ is an upper bound for $A$ and $y \in P$ is such that $x \preccurlyeq y$ and $x \neq y$, then $y \in U_{A}$. Let $U_{\varnothing}=\left\{x_{0}\right\}$, where $x_{0}$ is an arbitrary element of $P$. Let $g$ be a mapping with domain $X:=\left\{U_{A}: A \in \mathcal{T}\right\}$, and now, we define a mapping $g$ from $X$ into itself by $g(x)=x$, i.e., $g$ is the identity mapping. For each $A \in \mathcal{T}$ let $f(A)=A \cup\left\{g\left(U_{A}\right)\right\}$. By definition of $g$ and $U_{A}$ we have $a \preccurlyeq g\left(U_{A}\right)$ for all $a \in A$ and all $A \in \mathcal{T}_{0}$. It is now clear that $f(A) \in \mathcal{T}$ for all $A \in \mathcal{T}$ and hence $f$ maps $\mathcal{T}$ into itself.

We shall prove that $\mathcal{T}$, partially ordered by inclusion, and $f$ satisfy the conditions of (d). First we observe that $\varnothing \in \mathcal{T}$ and $\varnothing \in A$ for all $A \in \mathcal{T}$ so $\mathcal{T}$ satisfies condition (i) of (d). Next let $\mathcal{R}$ be a nonempty subfamily of $\mathcal{T}$ such that $\mathcal{R}$ is chain ordered by inclusion and let $A=\cup_{B \in \mathcal{R}} B$. Let $a, b \in A$. There are sets $C, D \in \mathcal{R}$ with $a \in C$ and $b \in D$. Since $\mathcal{R}$ is a chain ordered by inclusion either $C \subset D$ or $D \subset C$ and in either case we see that there is one set in $\mathcal{R}$ which contains both $a$ and $b$. Since each set in $\mathcal{R}$ is a chain ordered subset of $P$ it follows that either $a \preccurlyeq b$ or $b \preccurlyeq a$. This proves that $A \in \mathcal{T}$ and it is then easy to see that $A=\sup \mathcal{R}$. Thus $\mathcal{T}$ satisfies the condition of chain completeness of (d). By definition of $f$ we have $A \subset f(A)$. Also condition (ii) of (d) is satisfied. Also, it follows immediately that condition (iii) of (d) is satisfied.

We can now conclude from (d) that there is a set $A_{0} \in \mathcal{T}$ with $f\left(A_{0}\right)=A_{0}$. Thus we have a contradiction. The proof is now complete.

\section{An Illustration}

We notice that, by the application of Lemma 1a by Tasković [2004, p. 61] one can simultaneously obtain the upper and lower bounds of the roots of the following equation in the form

$$
\begin{aligned}
x^{n}= & a_{1} x^{n-1}+a_{2} x^{n-2}+\cdots+a_{n}, \\
& \left(a_{1}+\cdots+a_{n}>0 ; \quad a_{i} \geq 0(i=1, \ldots, n)\right) .
\end{aligned}
$$

Then, by Minimax Principle in Tasković [2004, Theorem 1a, p. 64], as an immediate consequence we obtain the following statement for solutions of algebraic equations of the preceding form. 
Theorem 5 (Tasković [2004]). A point $\xi \in \mathbb{R}_{+}:=(0,+\infty)$ is the root of the equation (AE) if and only if the following equality holds

$$
\begin{aligned}
\xi & :=\max _{\lambda_{2}, \ldots, \lambda_{n} \in \mathbb{R}_{+}} \min \left\{\lambda_{2}, \ldots, \lambda_{n}, a_{1}+\frac{a_{2}}{\lambda_{2}}+\cdots+\frac{a_{n}}{\lambda_{n}^{n-1}}\right\}= \\
& =\min _{\lambda_{2}, \ldots, \lambda_{n} \in \mathbb{R}_{+}} \max \left\{\lambda_{2}, \ldots, \lambda_{n}, a_{1}+\frac{a_{2}}{\lambda_{2}}+\cdots+\frac{a_{n}}{\lambda_{n}^{n-1}}\right\} .
\end{aligned}
$$

We notice that, if $0<a_{1}+\cdots+a_{n}<1$, then a root $\xi$ of the equation (AE) of the form (Ro) lie in the open interval $(0,1)$, i.e., $\xi \in(0,1)$, such that

$$
\begin{aligned}
\xi & =\max _{\lambda_{2}, \ldots, \lambda_{n} \in(0,1)} \min \left\{\lambda_{2}, \ldots, \lambda_{n}, a_{1}+\frac{a_{2}}{\lambda_{2}}+\cdots+\frac{a_{n}}{\lambda_{n}^{n-1}}\right\}= \\
& =\min _{\lambda_{2}, \ldots, \lambda_{n} \in(0,1)} \max \left\{\lambda_{2}, \ldots, \lambda_{n}, a_{1}+\frac{a_{2}}{\lambda_{2}}+\cdots+\frac{a_{n}}{\lambda_{n}^{n-1}}\right\} .
\end{aligned}
$$

From Theorem 4 (cases (b) and (c)), on the set $\mathcal{R}^{*}:=[0,+\infty]$ with the ordinary ordering $\leq$, in this case, applying Theorem 5 we have the following illustrations. We notice that, a maximal element $z_{u s}$ on the upper side $\mathcal{R}_{u s}^{*}$ of the set $\mathcal{R}^{*}$ is in the following form as

$$
z_{u s}=\min _{\lambda_{2}, \ldots, \lambda_{n} \in \mathbb{R}_{+}} \max \left\{\lambda_{2}, \ldots, \lambda_{n}, a_{1}+\frac{a_{2}}{\lambda_{2}}+\cdots+\frac{a_{n}}{\lambda_{n}^{n-1}}\right\} ;
$$

thus $z_{u s} \in \mathcal{R}_{u s}^{*}$ as the maximal element by (b) of Theorem 4 and as the fixed point of the mapping $f(x):=\max \left\{x, a_{1}+a_{2} / x+\cdots+a_{n} / x^{n-1}\right\}$ on the upper side $\mathcal{R}_{u s}^{*}$ of the set $\mathcal{R}^{*}$ by (c) of Theorem 4 . We notice that in this case for the function $f: \mathcal{R}^{*} \rightarrow \mathcal{R}^{*}$ the condition (M) holds, i.e., we have the following form of $(\mathrm{M})$ as

$$
x \leq \max \left\{x, a_{1}+a_{2} / x+\cdots+a_{n} / x^{n-1}\right\}:=f(x)
$$

for all $x \in \mathcal{R}^{*}$, and thus for all $x \in \operatorname{Sub} f\left(\mathcal{R}^{*}\right)$. Also, on the other hand, the point of the following form as

$$
z_{l s}=\max _{\lambda_{2}, \ldots, \lambda_{n} \in \mathbb{R}_{+}} \min \left\{\lambda_{2}, \ldots, \lambda_{n}, a_{1}+\frac{a_{2}}{\lambda_{2}}+\cdots+\frac{a_{n}}{\lambda_{n}^{n-1}}\right\}
$$

is a point on the lower side $\mathcal{R}_{l s}^{*}$ of the set $\mathcal{R}^{*}$, i.e., $z_{l s} \in \mathcal{R}_{l s}^{*}$ as the maximal element by (b) of Theorem 4 and as the fixed point of the mapping $f$ on the lower side $\mathcal{R}_{l s}^{*}$ of the set $\mathcal{R}^{*}$ by (c) of Theorem 4 .

In connection with this we can and the point $\xi$ given in (Ro) defined as third maximal element $\xi=z_{m s} \in \mathcal{R}_{m s}^{*}$ by (b) of Theorem 4 and as the fixed point of the mapping $f$ on the medial side $\mathcal{R}_{m s}^{*}$ of the set $\mathcal{R}^{*}$ by (c) of Theorem 4. 


\section{REFERENCES}

[1982] Moore, Gregory. H. Zermelo's axiom of choice - its origins, development and influence; Springer-Verlag, New York, Heidelberg, Berlin, Vol. 8, 1982, 410 pages.

[1985] Rubin, H., and Rubin, J. Equivalents of the Axiom of Choice, North-Holland Publ. Comp., Amsterdam-London, 1970, 134 p.p.; Equivalents of the Axiom of Choice-II, North-Holland, Publ. Comp. Amsterdam-New York-Oxford, 1985, 322 pages.

[1973] Jech, Thomas. J. The Axiom of Choice, Amsterdam, North-Holland, 1973.

[1998] Howard, Paul., and Rubin, Jean. E. Consequences of the axiom of choice, p.cm. - (Mathematical surveys and monographs, ISSN 0076-5376, vol. 59), Amer. Math. Soc. Providence, Rhode Island, USA, 1998, 432 pages.

[1975] Tasković, Milan. R. Fixed points of contractive and some other mappings, Ph These, University of Beograd, 1975, 93 pages.

[1979] Tasković, Milan. R. Banach's mappings of fixed points on spaces and ordered sets, These, Univesity of Beograd, 1978, 151 pages; Math. Balkanica, 9 (1979), 130 pages.

[1986] Tasković, Milan. R. On an equivalent of the axiom of choice and its applications, Math. Japonica, 31 (1986), 979-991.

[1988] Tasković, Milan. R. Characterizations of inductive posets with applications, Proc. Amer. Math. Soc., 104 (1988), 650-659.

[1992] Tasković, Milan. R. New maximal principles, Math. Japonica, 37 (1992), 549-554.

[1992] Tasković, Milan. R. The axiom of choice, fixed point theorems, and inductive ordered sets, Proc. Amer. Math. Soc., 116 (1992), 897-904.

[1993] Tasković, Milan. R. Nonlinear Functional Analysis, (Fundamental Elements of Theory). First Book: Monographs, Zavod za udžbenike i nastavna sredstva, Beograd 1993, 812 pages, (Serbo-Croation). English summary: Comments only new main results of this book, Vol. 1 (1993), 713-752.

[2001] Tasković, Milan. R. Nonlinear Functional Analysis, Second Book: Monographs - Global Convex Analysis - General convexity, Variational methods and Optimization, Zavod za udžbenike i nastavna sredstva and Vojnoizdavački zavod, Beograd 2001, (in Serbian), 1223 pages. English summary: Transversal and fixed points, forks, general convex functions, and applications, Vol. 2 (2001), 1067-1176.

[2004] Tasković, Milan. R. Transversal Sets, Math. Moravica, 8-2 (2004), 53-93.

[2005] Tasković, Milan. R. Theory of transversal point, spaces and forks, Monographs of a new mathematical theory, VIZ-Beograd 2005, (in Serbian), 1054 pages. English summary: 1001-1022.

Milan R. TAsković

FaCUlty of Mathematics

11000 Belgrade, P.O. Box 550

Serbia

Home Address:

Milan R. Tasković

Nehruova 236

11070 BELGRADE

SERBIA

E-mail address: andreja@predrag.us 